\title{
Utilización de screencasts para un aprendizaje activo
}

\author{
González-Siso, María-Isabel; Becerra Fernández, Manuel \\ Grupo EXPRELA, Facultade de Ciencias, Universidade da Coruña.
}

\section{RESUMEN}

La presente innovación consistió en la elaboración por los estudiantes de screencasts, es decir, vídeos cortos en los que se graba parte o la totalidad de la pantalla del ordenador, acompañados por audios con las explicaciones correspondientes, acerca de temas propuestos por los profesores de la materia. Se recomendó el formato PechaKucha: 20 diapositivas de 20 segundos cada una. Al finalizar la experiencia, los estudiantes respondieron un cuestionario en la plataforma de teleenseñanza Moodle. De las respuestas obtenidas destacamos: la mayoría necesitó menos de 2 horas para la elaboración del screencast; sólo el 5\% había utilizado antes este recurso; a todos los estudiantes la elaboración del screencast les ayudó a sintetizar los contenidos del tema; al 95\% les ayudó a preparar la presentación oral; sólo el 10\% optó por tener a su disposición los screencasts elaborados por sus compañeros para el estudio, prefiriendo resúmenes de los temas; el $80 \%$ opinó que 20 segundos por diapositiva era poco tiempo para desarrollar los contenidos; al 58\% le gustaría disponer de los temas impartidos por los profesores en formato vídeo; el $90 \%$ asistiría igualmente a las clases presenciales aun disponiendo de los vídeos, si bien la mitad con menor frecuencia.

PALABRAS CLAVE: Screencast, PechaKucha, aprendizaje activo 


\section{CITA RECOMENDADA:}

González-Siso, M.l.; Becerra Fernández, M. (2019): Utilización de screencasts para un aprendizaje activo. En De la Torre Fernández, E. (ed.) (2019). Contextos universitarios transformadores: construíndo espazos de aprendizaxe. III Xornadas de Innovación Docente. Cufie. Universidade da Coruña. A Coruña (pág. 15-24).

DOI capítulo: https://doi.org/10.17979/spudc.9788497497121.015

DOl libro: https://doi.org/10.17979/spudc.9788497497121

\section{ABSTRACT}

The present innovation consisted of the preparation by the students of screencasts, i.e. short videos recording the computer screen (full or partial) with the corresponding explanations in audio, about themes proposed by the professors. The PechaKucha format was suggested: 20 slides of 20 seconds each one. At the end of the experience, the students answered a questionnaire in Moodle. From the responses obtained we outline: the majority needed less than 2 hours to record the screencast; only $5 \%$ had used this resource previously; all the students considered the screencast usefull to synthesize the contents of the theme; $95 \%$ considered it usefull to prepare the oral presentation; only $10 \%$ chose the screencasts made by their colleagues to study the themes, being summaries the preferred option; $80 \%$ thought that 20 seconds per slide was insufficient time to develop the contents; $58 \%$ would appreciate to have available the lectures taught by the professors in video format; $90 \%$ would continue assisting to presential master classes, although half less often.

KEY WORDS: Screencast, PechaKucha, active learning 


\section{INTRODUCCIÓN}

En un mundo cada vez con mayor impacto de los estímulos audiovisuales y recursos multimedia, es de interés aplicar todas estas tecnologías al ámbito educativo. En este sentido, uno de los recursos multimedia que está adquiriendo mayor importancia como tecnología educativa es el uso de los screencast (Ghilay, Y., 2018). Un screencast no es más que un vídeo corto en el que se graba parte 0 la totalidad de la pantalla del ordenador, acompañado por un audio con las explicaciones correspondientes. Entre algunos de los ejemplos de screencast aplicados a la educación tenemos los tutoriales, video lecciones o presentaciones de diapositivas. El principal beneficio para los estudiantes es que les permite un aprendizaje personalizado ya que por una parte posibilita un acceso a la grabación en cualquier momento y por otra parte el estudiante puede hacer pausa y volver a ver lo que no ha entendido. Este recurso también ofrece ventajas para el docente ya que puede utilizarlo para explicar aquellas partes más complicadas de la materia o las que habitualmente el estudiante tiene más dudas, evitando por tanto las repeticiones tediosas (Mullamphy et al., 2010). Aunque el término screencast data del 2004 (Sugar et al., 2010), ya existía en los años noventa tecnología para grabar la pantalla del ordenador como el software de pago Lotus/BBM ScreenCam (http://smartguyz.com/) (Barra Arias, E., 2014). Actualmente, además del software mencionado anteriormente, hay una gran variedad de alternativas para realizar screencast bien de manera gratuita como Open Broadcaster Software (https://obsproject.com/es), de pago como TechSmith Camtasia (https://www.techsmith.com/video-editor.html), Adobe Captivate (https://www.adobe.com/es/products/captivate.html) o TouchCast Pitch dentro del paquete Microsoft (https://www.microsoft.com/es-es/p/touchcast-pitch/9p1bvglf68hw?activetab= pivot:overviewtab), o bien con versiones gratuitas y posibilidad de funciones más avanzadas mediante pago como $\begin{array}{llll}\text { Screencast-0-matic } \quad(\text { https://screencast-0-matic.com/) } & 0 & \text { FlashBack }\end{array}$ (https://www.flashbackrecorder.com/express/). La mayoría de estos programas permiten seleccionar si queremos introducir sólo audio o audio y video (en caso de tener una cámara 
webcam instalada), grabar toda la pantalla o una parte, resaltar el cursor y exportar y compartir fácilmente el vídeo.

Por otra parte, la técnica de presentación PechaKucha requiere de un proceso de concreción y síntesis para resaltar las ideas principales manteniendo la atención de la audiencia. Básicamente supone presentar un tema, trabajo, proyecto, idea 0 experiencia utilizando para ello únicamente 20 diapositivas de 20 segundos de duración cada una de ellas, usando la transición automática de diapositivas (la duración total de la exposición será por tanto de 6 minutos y 40segundos). Aunque inicialmente esta técnica fue creada en 2003 por dos arquitectos para promocionar su proyecto, rápidamente se ha extendido a otros ámbitos, incluido el educativo (https://www.pechakucha.org/). Esta modalidad de presentación fomenta competencias transversales en el estudiante, como la capacidad de síntesis y concreción, la creatividad, la comunicación oral y visual, además de potenciar el aprendizaje activo y autónomo (Botella Nicolás, A.M. et al., 2018).

En el presente trabajo se relata la experiencia de combinar ambas metodologías: screencast y PechaKucha dentro del ámbito docente. Se propuso a los estudiantes la elaboración de un screencast sobre un tema de la materia utilizando para ello el formato de presentación PechaKucha.

\section{DESCRIPCIÓN DE LA EXPERIENCIA}

La presente propuesta se desarrolló en una materia optativa (Fundamentos Bioquímicos de Biotecnología) del cuarto y último curso del grado en Biología de la Universidade da Coruña, durante el cuso académico 2017-18.

Los estudiantes debieron trabajar de una manera activa, en pareja 0 individualmente a su elección, sobre determinados temas que escogieron entre los propuestos por el profesorado de la materia y que, por formar parte del programa, fueron objeto de preguntas en el examen escrito de la asignatura. El valor de este trabajo fue el $20 \%$ de la calificación final de la asignatura. 
Además de exponer los temas oralmente con apoyo audiovisual ante el resto del grupo de clase, en un tiempo máximo de 20 minutos (15 de exposición más 5 de preguntas), como material de entrega tuvieron que realizar una presentación en formato screencast. De entre todos los programa de captura de vídeo, se recomendó Screencast-o-matic (https://screencast-o-matic.com/) por ser una herramienta sencilla de usar y que permite, en su versión gratuita, grabaciones de hasta quince minutos y exportar fácilmente el fichero de vídeo obtenido. Dicho fichero tiene un tamaño que permite manejarlo en la plataforma de teleenseñanza Moodle sin sobrecargar el espacio disponible online. Se recomendó, además, utilizar el formato PechaKucha.

Al finalizar la experiencia los estudiantes debieron responder a un cuestionario elaborado en la plataforma Moodle, en el que se les plantearon 17 preguntas de las cuales 12 eran para elegir entre varias opciones, mientras que 5 les permitían expresar su opinión redactando un pequeño texto.

\section{RESULTADOS}

Respondieron al cuestionario 19 estudiantes de un total de 25 matriculados en la asignatura. En general los estudiantes consideraron el recurso de elaboración de screencasts novedoso y útil para preparar la exposición de su trabajo. A continuación, se expone un análisis detallado de las respuestas obtenidas a las preguntas planteadas (Figura 1).

Sólo tres estudiantes hicieron el trabajo individualmente, el resto ( $84 \%$ del total) prefirieron hacerlo en grupo de dos personas. Cada estudiante debía presentar un PechaKucha (20 diapositivas por 20 segundos cada una) en cualquier caso.

En cuanto al tiempo empleado para la grabación del video, 10 estudiantes (53\% del total) necesitaron menos de dos horas, de los cuales la mayoría (42\%) necesitó de 1 a 2 horas.

Un 16\% de los estudiantes tuvieron dificultades con el programa de grabación recomendado (Screencast-0-matic) y optaron por buscar y utilizar otro programa alternativo. Entre ellos hubo quien, además, utilizó un segundo programa para editar el vídeo una vez grabado. A pesar de 
estar en último año del grado, sólo el 5\% (1 estudiante) manifestó haber utilizado previamente programas para grabar la pantalla del ordenador del tipo Screencast-0-matic.

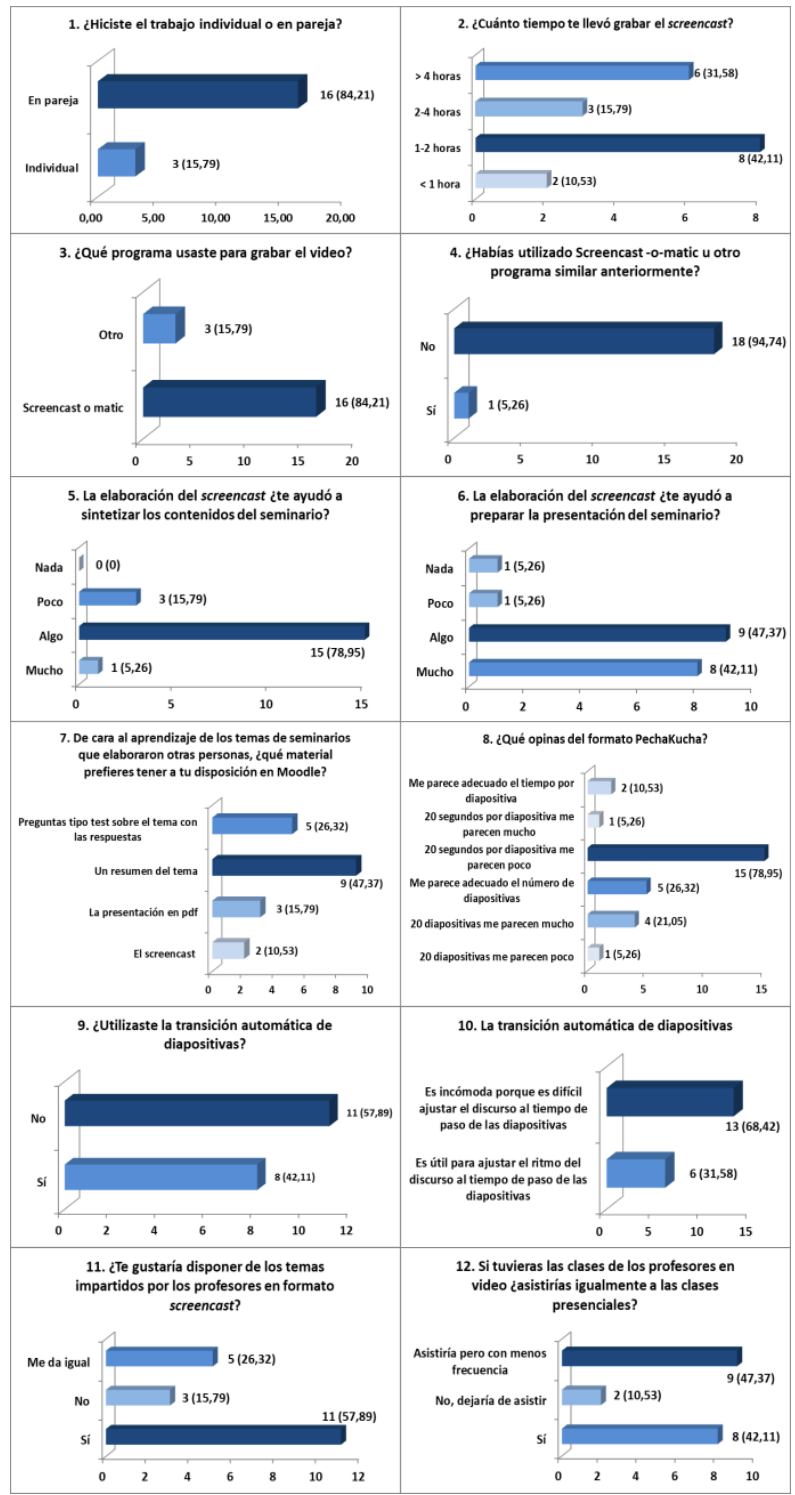

Figura 1: Preguntas realizadas a los estudiantes con sus respectivas respuestas. Se indica el número de estudiantes que respondió cada respuesta y en paréntesis se representa el porcentaje sobre el total de respuestas para cada una de las preguntas. 
Todos los estudiantes consideraron que la elaboración del screencast les ayudó a sintetizar los contenidos del tema en varios grados: $5 \%$ mucho, $79 \%$ algo, $16 \%$ poco.

Al 95\% de los estudiantes la elaboración del screencast les ayudó a preparar la exposición oral del tema: $42 \%$ mucho, $48 \%$ algo, $5 \%$ poco.

Sólo el $11 \%$ de los estudiantes optó por tener a su disposición los screencast elaborados por sus compañeros para el estudio/aprendizaje de los temas, prefiriendo el resto un resumen del tema (47\%), preguntas tipo test acerca del tema con las respuestas (26\%), o las diapositivas de la presentación en formato pdf (16\%).

El 79\% de los estudiantes opinó que el formato PechaKucha de 20 segundos por diapositiva era demasiado estricto y le pareció poco tiempo para las explicaciones. De hecho, entre las sugerencias de mejora planteadas por los estudiantes, predominaron las de aumentar el tiempo disponible por diapositiva, a expensas bien de aumentar el tiempo total de exposición 0 bien de reducir el número de diapositivas. Algunos también sugirieron reducir los contenidos de los temas para adaptarlos mejor al formato PechaKucha.

Del mismo modo, el 58\% de los estudiantes no usó la transición automática de diapositivas, manifestando el $68 \%$ que les resultaba incómoda.

El $58 \%$ de los estudiantes respondió que les gustaría disponer de los temas impartidos por los profesores en formato vídeo. En ese caso, es decir, aunque tuvieran las clases de los profesores en vídeo, sólo un $11 \%$ de estudiantes dejaría de asistir a las clases presenciales, el $89 \%$ restante seguiría asistiendo, pero el $47 \%$ probablemente lo haría con menor frecuencia. Los estudiantes que continuarían asistiendo a clase presencial, mayoritariamente lo harían para poder preguntar en directo y en el momento las dudas. A pesar de que se habilitó en Moodle un foro de preguntas y respuestas a modo de tutoría grupal y de que pudieron utilizar las tutorías presenciales y el correo electrónico, los estudiantes siguieron prefiriendo la interacción directa y espontánea en la clase presencial. Aunque a los vídeos les reconocen la ventaja de poder pausarlos y re-visualizarlos en cualquier horario, los materiales audiovisuales 
y telemáticos los consideran en general complementarios, pero no sustitutivos, a la clase magistral.

Finalmente, entre las sugerencias de mejora planteadas por los estudiantes, nos llamó la atención una referida a la preocupación porque los screencasts elaborados por los compañeros, y que usaron como material de estudio, pudieran contener errores, solicitando que los profesores los editaran o modificaran de algún modo para corregir dichos errores. En esa línea pensamos mejorar la experiencia aquí descrita durante el presente y los próximos cursos.

\section{CONCLUSIONES}

De la experiencia llevada a cabo se concluye que los screencast parecen ser una herramienta útil para fomentar el aprendizaje activo, así como un material de interés para los estudiantes. Mientras que el formato PechaKucha parece tener algunas limitaciones a la hora de desarrollar y comprender temas con cierta complejidad.

\section{REFERENCIAS}

Barra Arias, E. (2014). Nuevos métodos y herramientas para la creación y utilización de recursos multimedia en la educación. Tesis Doctoral. Universidad Politécnica de Madrid. http://oa.upm.es/30855/1/ENRIQUE_BARRA_ARIAS.pdf.

Botella Nicolás, A.M., Hurtado Soler, A. \& Ramos Ahijado, S. (2018). PechaKucha como herramienta de innovación educativa en el Espacio Europeo de Educación Superior. En REDINE (Ed.), Innovative strategies for Higher Education in Spain (pp. 201-212). Eindhoven, NL: Adaya Press.

Ghilay, Y. (2018). Video-based learning of quantitative courses in higher education. $i$ manager's Journal of Educational Technology, vol. 15, pp. 16-27.

Mullamphy, D., Higgins, P., Belward, S. \& Ward, L. M. (2010). To screencast or not to screencast. ANZIAM Journal, vol. 51, pp. C446-C460. 
Sugar, W., Brown, A. \& Luterbach, K. (2010). Examining the anatomy of a screencast: uncovering common elements and instructional strategies. International Review of Research in Open and Distance Learning, vol. 11, pp. 1-20. 
